\title{
Psychiatric disorders in menopause
} Dimitrios Kalfas*1, Thanos Didaskalou' ${ }^{1}$, Katerina Polyzoi ${ }^{1}$, Georgios Anthimidis ${ }^{1}$, Dimitrios Beis ${ }^{2}$, Presveia Tatsi ${ }^{1}$, Konstantinos Vasilakos ${ }^{1}$, Nikoletta Pampouchidou ${ }^{3}$, Ioannis Pavlidis ${ }^{4}$ and Abdel Tawfik ${ }^{1}$

Address: ${ }^{1}$ Giannitsa Gen Hospital, Pella, Greece, ${ }^{2}$ Katerini Mental Health Center, Greece, ${ }^{3}$ Mehaniona Health Center, Greece and ${ }^{4}$ I.K.A. Central Thessaloniki, Greece

* Corresponding author

from International Society on Brain and Behaviour: 2nd International Congress on Brain and Behaviour

Thessaloniki, Greece. 17-20 November 2005

Published: 28 February 2006

Annals of General Psychiatry 2006, 5(SuppI I):SI84 doi:I0.II86/I744-859X-5-SI-SI84

\section{Background}

The aim of this study was to examine the occurrence of psychological symptoms in women during menopause.

\section{Materials and methods}

The study was carried out with a sample of 117 menopausal women, who presented as outpatients to the psychiatric clinic of Giannitsa general hospital after gynecological referral. Their somatic complaints were recorded and clinical and demographic characteristics like age, family characteristics (number of children), cultural level, place of residence, (village or city), duration and conditions of menopause as well as circumstances of menopause presentation (normal or surgical) were determined. We studied life events and the period they occurred. A clinical psychiatric interview ensued along with completion of DSM (SCID) questionnaire. Psychometric methods like BDI (Beck Depression Inventory) and STAI (State, Trail, Anxiety Inventory) were used for diagnostic evaluation. Diagnosis was based on DSM IV.

\section{Results}

Significant symptoms were identified in 32 women $(27.4 \%)$. Some sort of anxiety distress was identified in 17 women $(53.1 \%)$. Depression was diagnosed in ten women $(31.3 \%)$, while paranoic psychosis was found in only one case $(3.1 \%)$. In four cases diagnosis was determined after a second attempt using DSM IV due to exacerbation of symptoms of preexisting personality distress.

\section{Discussion}

A high percentage of women in menopause $(27.4 \%)$ is associated with psychiatric symptomatology needing appropriate treatment.

\section{References}

I. Sajatovic M, et al.: Mental illness and menopause: a patient and family perspecive. J Gend Specif Med 2003, 6:3I-34.

2. Claudia P, et al.: Panic disorder in menopause: a case control study. Maturitas 2004, 48: 147-I54.

3. Bezicioglou I, et al.: Depression - anxiety and disability in the premenopausal and postmenopausal period. Turk Psikiyatri Derg 2004, I 5: 199-207.

4. Antonievic IA, et al.: On the role of menopause for sleep-endocrine alterations associated with major depression. Psychoneuroendocrinology 2003, 28:40 I-4I8. 